\title{
Lymphatic Pump Treatment Increases Thoracic Duct Lymph Flow in Conscious Dogs with Edema Due to Constriction of the Inferior Vena Cava
}

\author{
Parna Prajapati, M.B.B.S., Pankhil Shah, M.B.B.S., M.P.H.,, Hollis H. King, D.O., Ph.D., ${ }^{1}$ \\ Arthur G. Williams, Jr., B.S., Pratikkumar Desai, M.B.B.S., ${ }^{1}$ and H. Fred Downey, Ph.D.,
}

\begin{abstract}
Background: Osteopathic lymphatic pump treatments (LPT) are used to treat edema, but their direct effects on lymph flow have not been studied. In the current study, we examined the effects of LPT on lymph flow in the thoracic duct of instrumented conscious dogs in the presence of edema produced by constriction of the inferior vena cava (IVC).

Methods and Results: Six dogs were surgically instrumented with an ultrasonic flow transducer on the thoracic lymph duct and catheters in the descending thoracic aorta and in IVC. After postoperative recovery, lymph flow and hemodynamic variables were measured 1) pre-LPT, 2) during 4 min LPT, 3) post-LPT, in the absence and presence of edema produced by IVC constriction. This constriction increased abdominal girth from $60 \pm 2.6$ to $75 \pm 2.9 \mathrm{~cm}$. Before IVC constriction, LPT increased lymph flow $(P<0.05)$ from $1.9 \pm 0.2 \mathrm{ml} / \mathrm{min}$ to a maximum of $4.7 \pm 1.2 \mathrm{ml} / \mathrm{min}$, whereas after IVC constriction, LPT increased lymph flow $(P<0.05)$ from $7.9 \pm 2.2$ to a maximum of $11.7 \pm 2.2 \mathrm{ml} / \mathrm{min}$. The incremental lymph flow mobilized by $4 \mathrm{~min}$ of LPT (ie, the flow that exceeded $4 \mathrm{~min}$ of baseline flow), was $10.6 \mathrm{ml}$ after IVC constriction. This incremental flow was not significantly greater than that measured before IVC constriction.

Conclusions: Edema caused by IVC constriction markedly increased lymph flow in the thoracic duct. LPT increased thoracic duct lymph flow before and after IVC constriction. The lymph flow mobilized by $4 \mathrm{~min}$ of LPT in presence of edema was not significantly greater than that mobilized prior to edema.
\end{abstract}

\section{Introduction}

A NUMBER OF CONDITIONS AND DISEASES CAUSE EDEMA. Pharmacological agents that control hypertension and improve cardiac function, such as diuretics and angiotensinconverting enzyme inhibitors, often reduce edema. ${ }^{1}$ However, various nonpharmacological treatment modalities such as physical activity, early ambulation, pneumatic compression devices, and physiotherapy are often employed to reduce edema. ${ }^{2,3}$ Lymphatic pump treatments (LPT) are used by osteopathic physicians to increase lymph flow and, thus, to treat edema. ${ }^{4-6}$ Increasing lymph flow by LPT not only removes excessive tissue fluid from the interstitial space but also removes inflammatory mediators, toxins, and metabolic waste products. ${ }^{4}$ In addition, increased lymph flow augments transport of leukocytes to the blood circulation. ${ }^{7}$
We previously demonstrated that LPT increases thoracic duct lymph flow in conscious dogs under normal conditions and after acute expansion of the extracellular space. ${ }^{8}$ However, the effects of LPT on lymph flow have not previously been studied in an experimental model of lymphedema. In the current investigation, we examined the potential of LPT to increase the lymph flow in six dogs with lymphedema caused by chronic constriction of the inferior vena cava.

\section{Methods}

\section{Surgical procedures}

This study was approved by the Institutional Animal Care and Use Committee. Experiments were conducted in six healthy mongrel dogs ( 3 males and 3 females) weighing 22$32 \mathrm{~kg}$.

\footnotetext{
${ }^{1}$ Department of Integrative Physiology and ${ }^{2}$ Osteopathic Research Center, University of North Texas Health Science Center, Fort Worth, Texas.

This research was supported by NIH grant U19 AT002023. Drs. King and Downey received partial salary support from the Osteopathic Research Center.
} 
Acepromazine maleate $(0.03 \mathrm{mg} / \mathrm{kg}$, s.c.) was administered $30 \mathrm{~min}$ before the dog was anesthetized with sodium pentothal $(5 \mathrm{mg} / \mathrm{kg}$, i.v). After endotracheal intubation, the lungs were mechanically ventilated, and anesthesia was maintained with $1 \%-3 \%$ isoflurane gas in oxygen. Arterial $\mathrm{O}_{2}$ saturation, heart rate, and body temperature were monitored continuously.

Under sterile conditions, the chest was opened in the $4^{\text {th }}$ intercostal space. A pressure monitoring catheter (LAP17; $17 \mathrm{ga}, 50.6 \mathrm{~cm}$, Edwards Lifesciences) was inserted into the descending thoracic aorta, and secured with a purse string suture. An inflatable balloon occluder (OC12HD, $12 \mathrm{~mm}$ Vascular Occluder, In Vivo Metric ${ }^{\mathrm{TM}}$ ) was placed around the inferior vena cava (IVC) just distal to the right atrium, so that later the IVC could be partially constricted to elevate venous blood pressure distal to the IVC occluder. The pericardium was incised to expose the heart. A $15 \mathrm{G}$, Microbore ${ }^{\mathrm{TM}}$ catheter was inserted into the right atrial appendage and advanced into the inferior vena cava, so that its tip was beyond the balloon occluder. A $2.0 \mathrm{~mm}$ diameter, ultrasonic flow transducer (model 2SB; Transonic Systems Inc.) was placed around the thoracic lymph duct and secured to the surrounding tissue. ${ }^{9}$

The aortic and IVC catheters, the inflation tube for the balloon occluder, and the cable of the flow transducer were secured to the subcutaneous tissue and exteriorized through the skin between the scapulae. A tube was inserted into the thoracic cavity through the $6^{\text {th }}$ intercostal space for postoperative drainage of fluid. Buprenorphine hydrochloride $(0.03 \mathrm{mg} / \mathrm{kg}$. i.m.) was administered just before closure of the chest incision. The incision was closed in layers, and the skin was stapled. A triple antibiotic cream (Polymyxin B SulfateBacitracin Zinc-Neomycin Sulfate) was applied on the surgical wound. The chest was covered with an elastic stocking, and the catheters and the chest tube were secured by a nylon jacket (Alice King Chatham Medical Arts). Buprenorphine hydrochloride $(0.03 \mathrm{mg} / \mathrm{kg}$. i.m.) was administered $6-8 \mathrm{~h}$ postoperatively to reduce pain. On the first postoperative day, the chest tube was removed after any accumulated fluid had been evacuated. Orbifloxacin $(3 \mathrm{mg} / \mathrm{kg}$, po sid) and cotrimoxazole ( $30 \mathrm{mg} / \mathrm{kg}$, po sid) were administered daily for 10 days postoperatively to prevent infection.

\section{Measurements and experimental protocol}

The dogs were allowed to recover from the surgery for 8-10 days. During the experiment, blood pressure, heart rate, abdominal venous pressure, and thoracic duct lymph flow were recorded (Grass model 7D polygraph, Astro-Med) and digitally processed by a data acquisition system (EMKA; v. 1.8.9.18). The baseline pressure and thoracic lymph flow readings were recorded while the dog was comfortably standing in a canine support sling (Alice King Chatham Medical Arts) with all four feet resting on the base of the sling stand. The data acquisition software recorded mean hemodynamic data at $10 \mathrm{sec}$ intervals. These values were then averaged for each minute of the data collection period and tabulated for the duration of LPT and at 5 and $10 \mathrm{~min}$ postLPT. In four dogs, the effects of LPT prior to inflation of the IVC occluder were recorded 10-12 days after instrumentation. In all six dogs, the effects of LPT after inflation of the IVC occluder were recorded. The incremental lymph flow pro- duced by LPT was calculated by integrating differences between the flow measured during each minute of LPT and the pre-LPT baseline value.

Beginning on approximately the $12^{\text {th }}$ postoperative day, inflation of the balloon occluder on the IVC was adjusted to produce a sustained IVC pressure of $15-18 \mathrm{mmHg}$. This was accomplished by injecting a dextrose-glycerol solution $(50 \%$ dextrose mixed with glycerol, 1:1 ratio) into the balloon (through its exteriorized catheter), daily as needed to maintain the IVC pressure at the desired value. The balloon inflations were performed in $0.25 \mathrm{ml}$ increments. During each incremental inflation of the occluder, IVC and aortic pressures were monitored, and the inflation of the occluder was adjusted, so that mean IVC pressure did not rise above $18 \mathrm{mmHg}$ and mean aortic pressure did not fall below $90 \mathrm{mmHg}$. After 5-6 days, only occasionally were adjustments of balloon inflation required. This continuous constriction of the IVC caused accumulation of interstitial fluid and ascites, which increased the abdominal girth by approximately $25 \%$ by 10-14 days after initial constriction of the IVC. The occluder remained inflated for the remainder of the investigation, including the LPT sessions.

After stabilization of the elevated abdominal girth for 2-3 days, 4 min of LPT was performed daily for 4-8 days. Aortic and inferior vena caval pressures, heart rate, and thoracic duct lymph flow were recorded just prior to LPT, continuously during $4 \mathrm{~min}$ of LPT, and continuously for $10 \mathrm{~min}$ following cessation of LPT. Pre-LPT data were collected by the data acquisition system for $1.5 \mathrm{~min}$ prior to beginning LPT. These data were averaged and reported as baseline pre-LPT values. Data were collected for $1 \mathrm{~min}$ intervals for $4 \mathrm{~min}$ of LPT and for two consecutive $5 \mathrm{~min}$ intervals post-LPT; data were averaged and reported at the midpoint of each collection interval.

To perform canine LPT, the operator sat behind the dog and placed both hands just below the costal margin on the ventral abdomen. The operator moved his hands rhythmically forward and upward to compress the abdomen without lifting the dog's feet from the sling base. The frequency of abdominal compressions was adjusted, so that the abdominal recoil after a previous compression was followed by another compression, thus maintaining the continuity of compressions without any pause.

After the last LPT session, the IVC balloon occluder was completely deflated and hemodynamic parameters were monitored until the abdominal girth returned to the preexperimental size. At the conclusion of this study, the dogs were transferred to another protocol.

\section{Statistical analysis}

Data are presented as means \pm SE and are graphed at the midpoint of each minute of LPT and for 5 and $10 \mathrm{~min}$ postLPT. Data were summarized with Microsoft Excel ${ }^{\mathrm{TM}} 2007$ and statistically analyzed with GB-Stat software, version 10.0. The effects of LPT on thoracic duct lymph flow and hemodynamic variables before $(n=4)$ and after IVC constriction $(n=6)$ was analyzed by a two-factor Analysis of Variance (ANOVA): factor A, two levels (absence or presence of IVC constriction); factor B, seven levels (during rest, four observations during LPT, and two observations after LPT). A repeated measures analysis was applied to data from animals within each factor 
A level. When ANOVA detected significant effects $(P<0.05)$ for factor $A$ and/or factor B, a Student-Newman-Keuls multiple comparison test was performed to identify significant differences among specific means. Baseline, integrated, and incremental lymph flows produced by $4 \mathrm{~min}$ of LPT before $(n=4)$ and after $(n=6)$ IVC constriction were analyzed by unpaired $t$-test. Abdominal girths before and after IVC constriction $(n=6)$ were analyzed by paired $t$-test.

\section{Results}

\section{Abdominal girth as an index of edema}

IVC constriction caused abdominal girth to increase from $60 \pm 2.6$ to $75 \pm 2.9 \mathrm{~cm}(P<0.01)$. However, LPT after IVC constriction had no significant effect on abdominal girth.

\section{Effects of lymphatic pump treatment (LPT) on hemodynamic variables (Table 1)}

Before constriction of the IVC. LPT tended to cause small decreases in heart rate, with the percent decrease ranging from $4 \%$ to $10 \%$. There was no significant change in the mean aortic blood pressure during LPT. Throughout LPT, mean IVC blood pressure was elevated significantly $(P<0.05)$, with the percent increase in mean IVC pressure ranging from $170 \%$ to $202 \%$. The largest increase occurred during the second minute of LPT, during which the IVC pressure increased from pre-LPT baseline value of $4.6 \pm 1.4$ to $13.4 \pm 2.1 \mathrm{mmHg}(P<0.05)$.

After constriction of the IVC. LPT had no effect on heart rate but the mean aortic blood pressure was significantly elevated throughout LPT $(P<0.05)$. Mean IVC blood pressure was elevated throughout LPT $(P<0.05)$, with the percent increase ranging from $36 \%$ to $49 \%$. The largest increase in the mean IVC blood pressure was observed during the first minute of LPT, when IVC pressure increased from the preLPT baseline value of $17.3 \pm 0.8$ to $26 \pm 1.7 \mathrm{mmHg}(P<0.05)$.

\section{Effects of IVC constriction on hemodynamic variables (Table 1)}

During IVC constriction, heart rate at baseline was significantly lower than before constriction, but during LPT there was no significant difference between before and after IVC constriction values. IVC constriction caused significant decreases in mean aortic blood pressure at pre-LPT, first minute of LPT, and post-LPT. Due to the IVC constriction, mean IVC blood pressure was significantly increased at baseline, during LPT and recovery periods.

\section{Effects of lymphatic pump treatment (LPT) on thoracic duct lymph flow}

Before constriction of the IVC. Figure 1 illustrates lymph flow at pre-LPT baseline, during LPT, and at 5 and $10 \mathrm{~min}$ post-LPT. Lymph flow was significantly elevated at $1 \mathrm{~min}$ $(P<0.01)$ and at $2 \mathrm{~min}(P<0.05)$ of LPT and tended to be elevated throughout remainder of the $4-\mathrm{min}$ period of LPT. The greatest increase in lymph flow, 147\%, was observed during the first minute on LPT. The integrated lymph flow (Fig. 2) during $4 \mathrm{~min}$ of LPT was $15.0 \pm 3.2 \mathrm{ml}$. This was $7.4 \mathrm{ml}$ greater than the $4 \mathrm{~min}$ integrated baseline flow $(P<0.05$, compared to zero integrated lymph flow). Lymph flow 


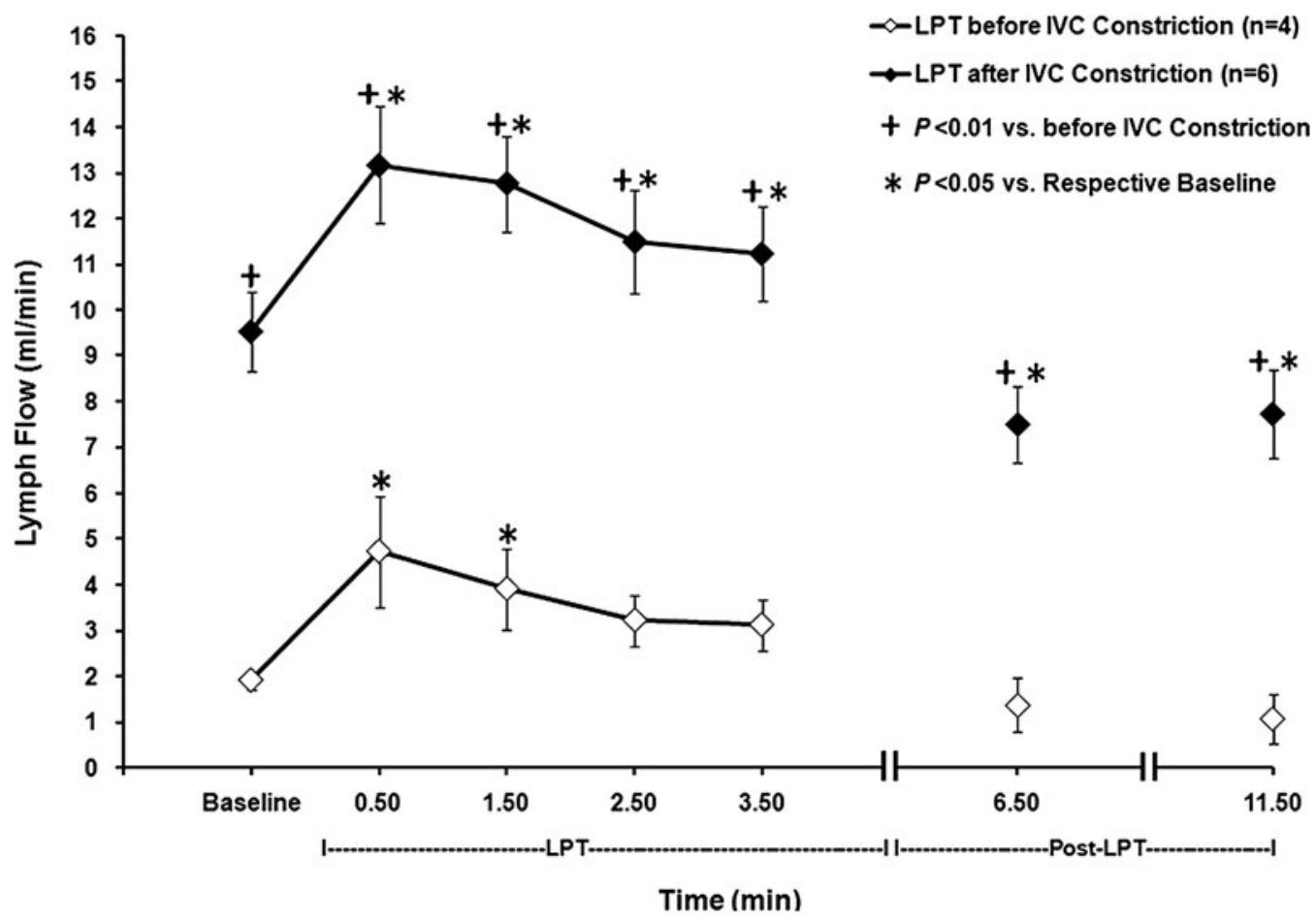

FIG. 1. Lymph flow (mean \pm S.E.) in the thoracic duct is plotted as a function of time before, during and after $4 \mathrm{~min}$ of lymphatic pump treatment (LPT). Data were collected before and after constriction of the inferior vena cava (IVC).

decreased rapidly after cessation of LPT, and did not differ significantly from the pre-LPT baseline value during $10 \mathrm{~min}$ post-LPT (Fig. 1).

After constriction of the IVC. The baseline lymph flow increased from $1.9 \pm 0.2 \mathrm{ml} / \mathrm{min}$ to $9.5 \pm 0.9 \mathrm{ml} / \mathrm{min}$ due to IVC constriction $(P<0.01 ;$ Fig. 1$)$. Compared to the pre-LPT baseline, lymph flow was elevated significantly throughout the 4 -min period of LPT $(P<0.05)$. The greatest increase in lymph flow, $38 \%$, was observed during the first minute of LPT. The integrated lymph flow (Fig. 2) during $4 \mathrm{~min}$ of LPT was $48.7 \pm 3.8 \mathrm{ml}$. This was $10.6 \mathrm{ml}$ greater than the $4 \mathrm{~min}$ integrated baseline flow ( $P<0.05$, compared to zero integrated lymph flow). Lymph flow decreased rapidly after cessation of LPT (Fig. 1), and was less $(19 \%-21 \%)$ than the pre-LPT baseline value for $10 \mathrm{~min}$ post-LPT $(P<0.05)$.

\section{Effects of IVC constriction on thoracic duct lymph flow}

IVC constriction markedly increased thoracic duct lymph flow at the pre-LPT baseline compared to the before IVC, preLPT baseline $(400 \%, P<0.01)$. During LPT, lymph flow was also markedly elevated compared to before IVC values $(178 \%-258 \%, P<0.01)$. Likewise, the post-LPT values were also elevated $(P<0.01)$. There was no significant interaction between factor A (absence or presence of IVC constriction) and factor B (observations during the experiment). Thus, LPT increased lymph flow independent of the IVC constriction, as is evident from Figure 1. The 4-min incremental flow (Fig. 2) observed in 6 dogs during LPT after IVC constriction $(10.6 \pm 3.2 \mathrm{ml})$ was not significantly greater than the incremental flow observed in 4 dogs during LPT before IVC constriction $(7.4 \pm 3.1 \mathrm{ml})$.

\section{Discussion}

A number of conditions and diseases cause edema. These include 1) heart failure and venous obstruction, conditions that increase capillary pressure; 2) toxins, infections, and burns, conditions that increase capillary permeability; 3) renal and hepatic diseases, conditions that decrease protein content of plasma; 4) surgery, infections, and cancer, conditions that block lymphatic drainage. ${ }^{10}$ Postoperative lymph stasis due to lack of mobility is also one of the most commonly encountered causes of edema. ${ }^{11,12}$ Edema is treated pharmacologically ${ }^{1}$ and also by physical maneuvers that increase lymph flow. ${ }^{4}$ Osteopathic physicians employ lymphatic pump treatments (LPT), which are manipulative therapies specifically designed to increase lymph flow. While it is generally thought that LPT would accelerate lymph flow from edematous tissue, there have been no direct measurements of lymph flow when LPT was performed in the setting of edema. Thus, this investigation examined the ability of LPT to augment thoracic duct lymph flow in instrumented dogs with edema produced by IVC constriction.

The major findings of this investigation were 1) a marked increase in thoracic duct lymph flow in the presence of edema; 2) LPT augmented this already increased lymph flow. Results also confirmed earlier reports that LPT augments lymph flow in the normal, nonedematous state.

Lymph flow is a function of the difference in peripheral tissue pressure and central venous pressure. ${ }^{10}$ As interstitial fluid volume increases during edema, tissue pressure also increases, and lymph flow increases. ${ }^{10}$ In fact, the lymphatic system has a large flow reserve, ${ }^{10}$ and this contributes to the "margin of safety" that initially may prevent edema when capillary filtration is increased. ${ }^{13}$ In the current investigation, 


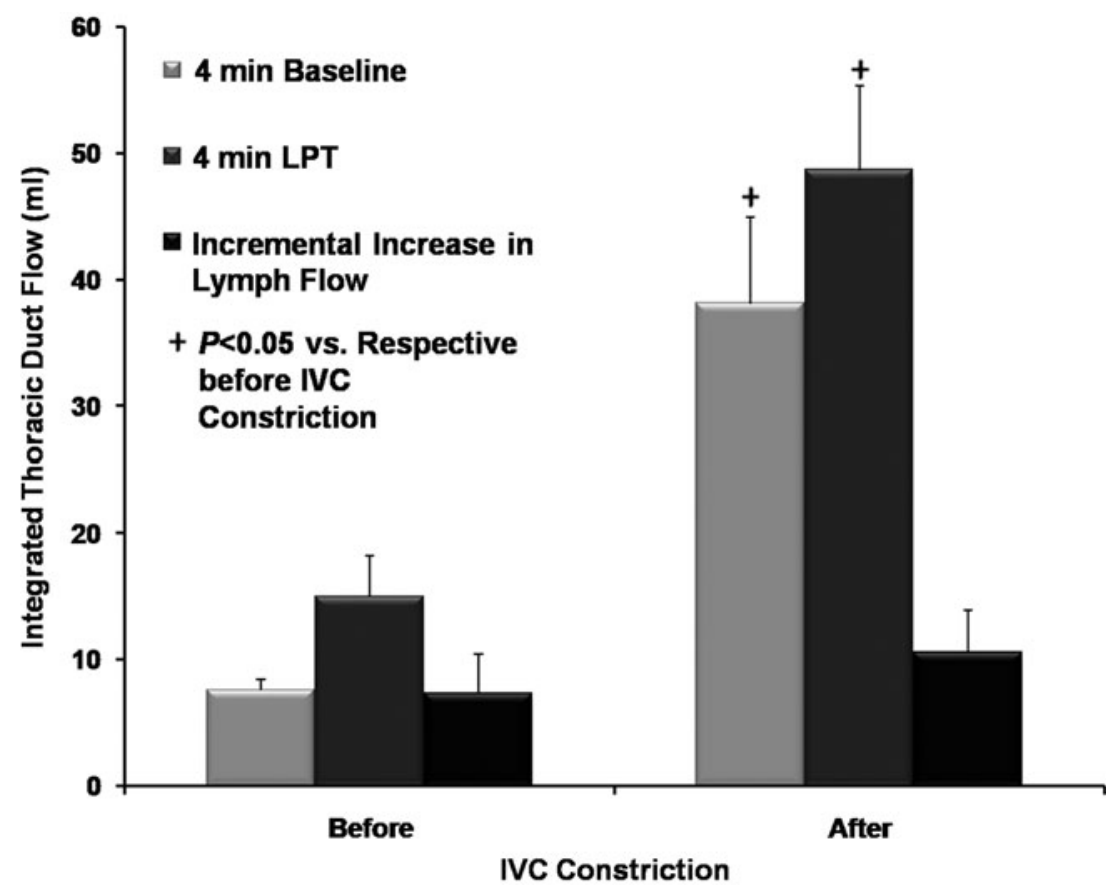

FIG. 2. Integrated lymph flows for $4 \mathrm{~min}$ of lymphatic pump treatment (LPT) before and after inferior vena cava (IVC) constriction are illustrated and compared with 4 min of resting, baseline flows. Data are mean \pm S.E.

the IVC constriction overwhelmed this margin of safety, and significant edema was created, as reflected by $25 \%$ gain in abdominal girth of the subjects. As would be expected, the baseline pre-LPT flow in the thoracic lymph duct was greatly elevated in this edematous state to $9.5 \pm 0.9 \mathrm{ml} / \mathrm{min}$, a $400 \%$ increase from the corresponding value prior to IVC constriction. In an earlier, related study, ${ }^{9}$ we acutely expanded the extracellular fluid by intravenous infusion of normal saline solution. The resulting elevation in capillary filtration caused a $182 \%$ increase in thoracic duct flow. Thus, it appears that the expansion of the interstitial space in the current investigation was somewhat greater than in the acute study. Also, intravenous infusion of saline would have diluted proteins in interstitial fluid and lymph, thereby decreasing the viscosity of the lymph, and this would have contributed to the elevated flow. This complication was avoided in the current study, but still the baseline lymph flow was greater. In either of these conditions, the role of increased interstitial pressure in accelerating lymph flow was apparent. This is confirmation of the importance of passive noncontractile forces in generating lymph flow. ${ }^{10}$

Rhythmic manual compressions of the chest, abdomen, or extremities to increase lymph flow are referred to as lymphatic pump treatments (LPT) in the osteopathic medical profession. These treatments are administered to treat edema, as well as to cleanse the interstitial space and to transport leukocytes and other immunologically important cells to the blood. ${ }^{7}$ In previous investigations with instrumented conscious dogs, we have demonstrated that LPT do, indeed, increase flow in the thoracic duct, and also increase leukocyte count in the lymph. ${ }^{7}$ In the current study, we confirmed that LPT does increase thoracic duct lymph flow in the normal nonedematous state, and then we demonstrated that in the edematous condition, LPT produced a further increase in lymph flow (Fig. 1). Clearly, in the edematous state there remained some lymph flow reserve that could be mobilized by the changes in interstitial pressure caused by LPT. However, the incremental increase in lymph flow above the pretreatment baseline flow, $10.6 \mathrm{ml}$, seems small considering the large increase in abdominal girth of these animals. Thus, it is not surprising that we detected no change in abdominal girth following LPT. In this investigation, only one LPT was performed per day. More frequent applications of LPT would be expected to have an additive effect on fluid mobilization. Also, in future studies, it would be informative to monitor urinary output when edema is treated by LPT.

In conclusion, this investigation demonstrated that edema produced by elevated inferior vena cava pressure greatly increased flow in the thoracic lymph duct of conscious, instrumented dogs. Lymphatic pump treatment further augmented this flow. However, the additional flow generated by $4 \mathrm{~min}$ of this treatment was not large.

\section{Acknowledgments}

The expert technical assistant of Linda Howard is gratefully acknowledged.

\section{Author Disclosure Statement}

The authors have no conflicts of interest or financial ties to disclose.

\section{References}

1. Kasper DL, Braunwald E, Hauser S, Longo D, Jameson LJ, Fauci AS. Edema. In: Harrison's Principles of Internal Medicine, $16^{\text {th }}$ Edition, McGraw-Hill, 2005: p. 214. 
2. Ho CKL, Sun MP, Au TWK, Chiu CS.W. Pneumatic pump reduces leg wound complications in cardiac patients. Asian Cardiovasc Thorac Ann 2006;13:452-457.

3. Partsch H. Intermittent pneumatic compression in immobile patients. Int Wound 2008;5:389-397.

4. Wallace E, McPartland JM, Jones III JM, Kuchera WA, Buser BR. Foundations for osteopathic medicine, $2^{\text {nd }} \mathrm{ed}$. East Lansing: Lippincott Williams \& Wilkins 2003: pp. 1061-1062.

5. Lane K, Worsley D, McKenzie D. Exercise and the lymphatic system: Implications for breast-cancer survivors. Sports Med 2005;35:461-471.

6. Bruno CJ. Manual techniques addressing the Lymphatic System: Origins and Development. American Osteopathic Association, J Am Osteopath Assoc 2005;105:457-464.

7. Hodge LM, King HH, Williams AG Jr, et al. Abdominal lymphatic pump treatment increases leukocyte count and flux in thoracic duct lymph. Lymphat Res Biol 2007;5:127133.

8. Knott ME, Tune JD, Stoll ST, Downey HF. Increased lymphatic flow in the thoracic duct during manipulative intervention. J Am Osteopath Assoc 2005;105:447-456.

9. Downey HF, Durgam P, Williams AG Jr, Rajmane A, King $\mathrm{HH}$, Stoll ST. Lymph flow in the thoracic duct of conscious dogs during lymphatic pump treatment, exercise, and ex- pansion of extracellular fluid volume. Lymphat Res Biol 2008;6:3-13.

10. Guyton AC, Hall JE. The microcirculation and the lymphatic system: Capillary fluid exchange, interstitial fluid, and lymph flow. In: Textbook of Medical Physiology, $10^{\text {th }}$ Edition, Philadelphia: W. B. Saunders Company, 2000: pp. 83-197.

11. Serazhitdinov AS, Fokin AA, Orekhova LA. [The treatment of varicosity associated with persistent edema of the lower limbs], Angiol Sosud Khir 2004;10:115-119, Russian.

12. Faenza A, Ferraro M, Gigli M, Paolis MD, Errani C, Mercuri M. Vascular homografts for vessel substitution in skeletal and soft tissue sarcomas of the limbs. Transplant Proc 2005;37:2692-2693.

13. Carl R, Honig M. Modern Cardiovascular Physiology, $1^{\text {st }}$ Edition. Boston: Little, Brown and Company, Inc., 1981: p. 176.

Address correspondence to: H. Fred Downey, Ph.D.

Department of Integrative Physiology University of North Texas Health Science Center 3500 Camp Bowie Blvd. Fort Worth, TX 76107

E-mail: fred.downey@unthsc.edu 